\title{
A Model for Kinetically Controlled Internal Phase Segregation During Aerosol Coagulation
}

\author{
H. Struchtrup, M.Luskin ${ }^{\dagger}$ and M.R. Zachariah \\ University of Minnesota, Minneapolis, MN 55455
}

August 25, 2000

\begin{abstract}
In previous studies of particle growth, we have synthesized binary metal oxide aerosols and have observed the evolution of internal phase segregation during growth of molten nanodroplets. We describe a new formulation of the aerosol general dynamic equation (GDE) that incorporates the phase segregation in a binary aerosol. The model assumes that complete phase segregation is the thermodynamically favored state, that no thermodynamic activation energy exists, and that the segregation process is kinetically controlled. We develop a GDE formulation that involves the solution of a distribution function $N_{n}(V)$, where $N_{n}(V)$ is the number density of aerosols with volume $V$ and $n$ phase domains (which we might think of as enclosures). The GDE is solved using a 2-D sectional model and under the assumption that the phase coalescence of the minority phase is controlled by Brownian coagulation. For the purposes of these initial studies, the rate laws governing the enclosures (minority phase) assume a monodisperse particle size distribution. The dynamical behavior of such a system is presented.
\end{abstract}

\section{Introduction}

The evolution of an aerosol population is described by a master equation, the "aerosol general dynamic equation" (GDE). The GDE has been employed to characterize the behavior in time and space of the particle size distribution function, which can include all the driving forces for particle growth (nucleation, surface growth, coagulation/coalescence, transport) [7].

*Institute for Mathematics and its Applications, struchtr@ima.umn.edu

${ }^{\dagger}$ School of Mathematics, luskin@math.umn.edu

${ }^{\ddagger}$ Particle Technology Laboratory, mrz@me.umn.edu: to whom correspondence should be addressed 
However, in these multi-component studies it is assumed that the components comprising the particles are homogeneously distributed within the particle. We have been involved in a number of multi-component aerosol dynamics studies in which the internal structure of the components within the aerosol particle are at the heart of our interest in the materials, their properties and function. For example, we have conducted studies on the formation of binary metal oxide systems with application to removal of heavy metals [2][3] as well as the formation of materials with novel and interesting properties [4][5][6].

In this study, we focus on one particular binary system $\left(\mathrm{SiO}_{2} / \mathrm{Fe}_{2} \mathrm{O}_{3}\right)$ which we have studied in considerable detail. For this iron oxide/silica system, our initial goal was to develop a method for producing a ferromagnetic cluster within a non-magnetic host, and the iron oxide/silica system was chosen because the phase behavior at very high temperatures, that is when both components are in the liquid state, indicated that the liquids would be immiscible.

Our initial success in producing these materials [13] indicated that further research into the mechanistic aspects of the growth was warranted. In subsequent studies, we employed both in-situ interrogation into the formation process [11], multi-component aerosol dynamic modeling [1], and molecular dynamics computation [14]. One of the primary conclusions from these works was that at these high temperatures, where the nanodroplets are in a liquid like state, the phase segregation taking place within the nanodroplet was probably driven by a kinetically limited transport within the nanodroplet. Figure 1 shows TEM images of these nanocomposites at two different growth times. The images make clear the existence of dark $\left(\mathrm{Fe}_{2} \mathrm{O}_{3}\right)$ and light $\left(\mathrm{SiO}_{2}\right)$ domains, in which with increasing time we see the growth of the iron oxide phase. One can imagine that the temporal evolution of the aerosol phase follows the general characteristics illustrated in Fig 2.

Our goal in this paper is to develop a new formulation of the GDE which characterizes both aerosol growth, and the evolution of the internal morphology of multi-component liquid aerosols in which the phases are immiscible.

\section{The Model}

Due to surface tension, the iron oxide phase forms sphere-like enclosures inside the aerosol droplets. Obviously, it is not possible to describe the individual enclosures inside individual droplets since the numerical efforts would be tremendous. Therefore, we supplement the usual statistical formulation for the droplets, as given by the GDE, with terms which account for the statistics of the iron oxide enclosures.

The enclosures are considered as an aerosol inside the droplet, where coagulation takes place due to the Brownian motion of the enclosures. In this first approach to the problem, we only account for the expected number of enclosures inside the droplets. Thus, we ignore 
most details of the physics inside the droplet. In particular, we do not account for the size distribution of the enclosures.

Furthermore, to make the initial development of the model more tractable, we assume that the characteristic time for nucleation is considerably shorter than the subsequent growth processes. Implicit is that both components do not have significantly different nucleation rates. These latter points are quite reasonable based on our prior in-situ and modeling studies (see references above). Under such conditions, we assume that we have no gas-solid material transfer, so that the only physical/chemical processes occurring are inter- and intraaerosol transfer processes. Finally, we also assume that the solubility of either component in the other is so small that enclosures cannot loose material by dissolution processes.

We consider aerosol droplets which consists of two immiscible components in a constant volume ratio, $c$. Our objective is the determination of the distribution of droplet volumes in time and the internal state of the droplet, that is, the number and size of enclosures in the droplets.

For this first paper, we restrict ourselves to the simplest case and ask for the number density $N_{n}(t, V) d V$ of droplets with volumes in $(V, V+d V)$ and $n$ enclosures at time $t$. In particular, we ignore the size distribution of enclosures, which therefore in a droplet of volume $V$ with $n$ enclosure have the volume $c V / n$. For simplicity, we shall write $N_{n}(t, V)$ as the number density distribution as a function of time.

The number density of particles with volume $V$ and any number of enclosures is given by

$$
\mathcal{N}(t, V)=\sum_{n=1}^{\infty} N_{n}(t, V)
$$

The theory for this quantity is well developed, and one finds an evolution equation for $\mathcal{N}$ which reads [7]

$$
\begin{aligned}
\frac{d \mathcal{N}(t, V)}{d t}=\frac{1}{2} & \int_{0}^{V} \sigma(U, V-U) \mathcal{N}(t, U) \mathcal{N}(t, V-U) d U \\
& -\mathcal{N}(t, V) \int_{0}^{\infty} \sigma(V, U) \mathcal{N}(t, U) d U
\end{aligned}
$$

Here, $\sigma(V, U)$ is the collision probability for droplets with the volumes $V$ and $U$. Assuming that no friction forces are exerted on the particles during their flight and that their velocities are distributed according to the Maxwell distribution, one finds $\sigma$ as [12]

$$
\sigma(V, U)=\left(\frac{3}{4 \pi}\right)^{1 / 6}\left(\frac{6 k T}{\varrho}\right)^{1 / 2}\left(\frac{1}{V}+\frac{1}{U}\right)^{1 / 2}\left(V^{1 / 3}+U^{1 / 3}\right)^{2}
$$

where $k$ denotes Boltzmann's constant, $T$ is the temperature and $\varrho$ is the mass density of the droplets. The first term of the right hand side of (2) accounts for the gain of particles with 
volume $V$ due to agglomeration of particles with volumes $U$ and $V-U$, while the second accounts for the loss due to agglomeration of droplets with volume $V$ and droplets with any other volume.

We shall now construct the corresponding equation for $N_{n}(t, V)$. Under the natural assumption that the collision probability $\sigma(V, U)$ does not depend on the number of enclosures, we have (time dependence suppressed in the notation)

$$
\begin{aligned}
\frac{d N_{n}(V)}{d t}=\frac{1}{2} & \int_{0}^{V} \sigma(U, V-U) \sum_{m=1}^{n-1} N_{m}(U) N_{n-m}(V-U) d U \\
& -N_{n}(V) \int_{0}^{\infty} \sigma(V, U) \sum_{m=1}^{\infty} N_{m}(U) d U \\
& +\sum_{m=n+1}^{\infty} \gamma_{m \rightarrow n}(V) N_{m}(V)-\sum_{m=1}^{n-1} \gamma_{n \rightarrow m}(V) N_{n}(V) .
\end{aligned}
$$

Here, again, the first two terms account for the gain and loss due to the agglomeration of droplets, while the last two terms refer to the gain and loss due to the coagulation of enclosures inside a droplet of volume $V$. The quantity $\gamma_{n \rightarrow m}(V) d t$ denotes the probability that in a droplet of volume $V$ the number of enclosures will change from $n$ to $m$ during the time $d t$; this quantity will be considered in the next section.

The coagulation equation (3) has two conservative properties:

1. The coagulation of enclosures does not change the total number $\mathcal{N}(t, V)$ as defined in (1), so that the summation of (3) over all $n$ gives the proper equation (2) for $\mathcal{N}(t, V)$. The simple proof relies on the fact that one can invert the sequence of summation as

$$
\sum_{n=1}^{\infty} \sum_{m=1}^{n-1}=\sum_{m=1}^{\infty} \sum_{n=m+1}^{\infty}
$$

2. The total volume density

$$
v_{\text {tot }}(t)=\sum_{n} \int V N_{n}(t, V) d V=\int V \mathcal{N}(t, V) d V
$$

is constant in time, that is, $v_{\text {tot }}(t)=v_{\text {tot }}(0)=$ constant. This is readily shown by multiplication of (2) with the volume $V$ and subsequent integration.

\section{Collision probability for enclosures}

\subsection{Mean time for agglomeration}

By definition, $1 / \gamma_{n \rightarrow m}(V)$ is the mean time for the change of the number of enclosures from $n$ to $m$ in a droplet of size $V$. For the calculation of this time, we assume that Brownian 
motion of the enclosures is the dominant physical process inside the droplet. Other possible growth mechanisms, such as Oswald ripening, are considered to play no important role. Moreover, in our simple model we do not account for the different volumes of the enclosures which are assumed to be spherical.

The theory of the coagulation of spherical particles of the same size provides a differential equation for their number density $\nu=n / V$ which reads [8]

$$
\frac{d \nu}{d t}=-K_{0} \nu^{2} \quad \text { with } \quad K_{0}=\kappa \frac{k T}{\eta},
$$

where $\eta$ denotes the viscosity of the surrounding medium (the silica in our case) and $\kappa$ is a number that for the case of rigid particles moving in a gas [8] has the value $\kappa=\frac{4}{3}$. In our case, however, the deformable enclosures move in a liquid. Moreover, equation (5) does not consider the finite size of the droplet and requires that the mean distance between enclosures is considerably smaller than the radius of the droplet. We assume that all aberrations from the ideal conditions for the validity of (5) can be absorbed into the number, $\kappa$. Thus, we have to consider $\kappa$ as a quantity to be determined by experiments. Nevertheless, due to lack of experimental data, we shall set $\kappa=\frac{4}{3}$.

The right hand side of (5) denotes the rate of change of the number density. The probability to find two particles at the same place is proportional to the square of the number density, and thus the rate of change must be proportional to $\nu^{2}$. Moreover, the increase of the temperature increases the movement of the particles due to Brownian motion while a higher viscosity acts against any movement. This explains the factor $k T / \eta$ in the rate of change.

Equation (5) can be integrated easily and the solution reads after introducing the particle number $\nu=n / V$

$$
n=\frac{n_{0}}{1+\frac{K_{0}}{V} n_{0} t},
$$

where $n_{0}$ is the number of enclosures at time $t=0$. It follows that the inverse mean time for the agglomeration of $m$ enclosures to $n$ enclosures in a droplet of volume $V$, that is, the desired quantity $\gamma$, is given by

$$
\frac{1}{t_{m \rightarrow n}}=\gamma_{m \rightarrow n}(V)=\frac{K_{0}}{V} \frac{n m}{m-n} .
$$

Thus, the probability of agglomeration increases with increasing $K_{0}$ (increasing temperature, decreasing viscosity) and decreases with increasing volume $V$ (increasing mean distance between particles). Moreover, large changes in the number of enclosures are less probable. Note that $\gamma$ is only needed in (3) for $n \neq m$, so that it is always finite.

It is remarkable that $\gamma_{m \rightarrow n}(V)$ depends only on the number of enclosures $n$, but not on their volume $c V / n$. Indeed, the concentration $c$ will not appear in the sequel. This is due to the simple model for the coagulation of the enclosures and will change if a more realistic model is used. 


\subsection{Coagulation of enclosures alone}

In order to get a glance on the behavior of the enclosures, we consider collision-free droplets of volume $V=V_{0}$. In (3), we set $N_{n}(V)=N_{n} \delta\left(V-V_{0}\right)$ and $\sigma=0$ (where $\delta(V)$ is the Dirac delta function) and obtain with (6)

$$
\frac{d N_{n}}{d t}=\sum_{m=n+1}^{\infty} \frac{K_{0}}{V_{0}} \frac{n m}{m-n} N_{m}-\sum_{m=1}^{n-1} \frac{K_{0}}{V_{0}} \frac{n m}{n-m} N_{n} .
$$

In actual numerical calculations, we have to restrict the maximum number of enclosures to some finite value, $n_{\max }$. For convenience, we introduce the dimensionless time $\hat{t}=t K_{0} / V_{0}$ so that the equation for coagulation of enclosures can be written as

$$
\frac{d N_{n}}{d \hat{t}}=\sum_{m=1}^{n_{\max }} \mathcal{A}_{n m} N_{m}, \quad n=1,2, \ldots, n_{\max } .
$$

The matrix $\mathcal{A}_{n m}$ is given by

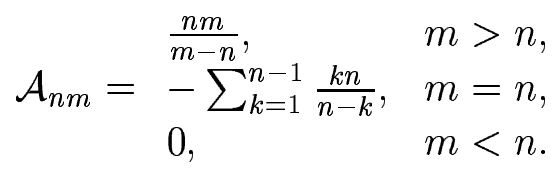

It should be noted that the variable $N_{n_{\max }}$ will always decrease with a characteristic time given by $1 / \omega_{n_{\max }}$ where $\omega_{n}=\sum_{k=1}^{n-1} \frac{k n}{n-k}$, while the number $N_{1}$ will always grow since $\omega_{1}=0$. Due to the conservation of the number density, we have for $n_{\max } \geq m$ that

$$
\sum_{n=1}^{n_{\max }} \mathcal{A}_{n m}=\sum_{n=1}^{m} \mathcal{A}_{n m}=0
$$

In principle, the set (8) of coupled ODE's can be solved by transformation to the principal axes. In practice, however, the large number of equations makes the transformation impossible, and we shall rely on numerical solutions.

The eigenvalues of $\mathcal{A}$ determine the characteristic time scales of the equations, and, due to the simple structure of the matrix, it is easy to state that these are given by the quantities $\omega_{n}$, that is, the rates of decrease of the number densities $N_{n}$. Obviously, the characteristic time scales cover a wide range of values: the number of droplets with a large number of enclosures will decrease much faster than the number of droplets with few enclosures.

This behavior allows for an adaptation of the time step during the numerical calculation. The initial time step $\delta \hat{t}$ is defined by the fastest scale,

$$
\delta \hat{t}=a / \omega_{n_{\max }}
$$


where $a<1$ is an appropriate constant; for our calculations we chose $a=0.02$. The number $N_{n_{\max }}$ will decrease and after several time steps its value will fall below a threshold $\Delta$, that is,

$$
N_{n_{\max }}<\Delta \bar{N} \quad \text { with } \quad \bar{N}=\frac{N_{\text {tot }}}{n_{\max }} \quad, \quad N_{\text {tot }}=\sum_{n=1}^{n_{\max }} N_{n} ;
$$

here we chose $\Delta=0.05$. If this condition is met, the number density $N_{n_{\max }}$ will not contribute significantly to the future process and will be ignored, that is, the corresponding equation will be canceled. Thus, there is not much change during the corresponding time scale $1 / \omega_{n_{\max }}$, while the relevant time scale is now given by $\delta \hat{t}_{1}=a / \omega_{n_{\max }-1}$. The calculation proceeds until $N_{n_{\max }-1}$ falls below the threshold, $N_{n_{\max }-1}<\Delta \bar{N}$. Then, again, the equation for $N_{n_{\max -1}}$ is ignored, and the new time scale is given by $\delta \hat{t}_{2}=a / \omega_{n_{\max }-2}$.

This procedure is carried on until $N_{2}<\Delta \bar{N}$, which corresponds to the stationary state, where all droplets have only one enclosure, $N_{1}=N_{\text {tot }}$. With this scheme, the number density of droplets with $n$ enclosures $(n \geq 2)$ is not equal to zero in the stationary state, but given by $N_{n}^{\text {stat }} \simeq \Delta \bar{N}, n \geq 2$ and $N_{1}^{\text {stat }}=(1-\Delta) N_{\text {tot }}$. Thus, $\Delta$ is directly related to the error that is introduced by the method.

Figure 3 shows the temporal development of $N_{n}$ for the normalized initial condition $N_{n_{\max }}(\tau=0)=$ $1, N_{n}(\tau=0)=0\left(n<n_{\max }\right)$ with $n_{\max }=2000$ (in arbitrary units). The pictures were taken in intervals of 600 time steps. Note that the actual time between the pictures almost doubles, due to the increase of the time step.

\subsection{An estimate for the maximum number of enclosures $n_{\max }$}

The question of what number $n_{\max }$ we have to chose is closely related to the time scales of the process. For the following, we shall assume that the relevant time scale is given by the mean free time of the droplets. The mean free time is defined as the average time a droplet travels before collision. With the mean volume $\bar{V}=\frac{4 \pi}{3} \bar{r}^{3}$ of the droplets and a total number density $N_{t o t}$, the mean free time of the droplets can be approximated as

$$
\tau_{D}=\frac{1}{\sigma(\bar{V}, \bar{V}) N_{t o t}}
$$

With $\bar{r}=5 \cdot 10^{-9} \mathrm{~m}$ and $N_{\text {tot }}=10^{18} \mathrm{~m}^{-3}$ we obtain for silica $\left(\varrho \simeq 2000 \mathrm{~kg} / \mathrm{m}^{3}\right)$ at $T=2000 \mathrm{~K}$ that

$$
\tau_{D} \simeq 2.74710^{-4} \mathrm{~s}
$$

We assume that processes on faster scales cannot be measured or are not of interest, respectively. Thus, only those characteristic times $\tau_{n}$ are important for the simulation of the 
enclosures which are of the same order of magnitude or larger, that is,

$$
\tau_{n}=\frac{V}{K_{0}} \frac{1}{\omega_{n}} \geq \tau_{D}, \quad n=2,3, \ldots
$$

The fastest scale is given by the characteristic time $\tau_{n_{\max }}$ and thus $n_{\max }$ follows as the solution of

$$
\tau_{n_{\max }}=\tau_{D}
$$

Figure 4 shows the characteristic times $\tau_{n}$ computed for silica at $T=2000 \mathrm{~K}\left(\eta \simeq 5.510^{6} \mathrm{~kg} / \mathrm{m} \mathrm{s}\right)$ as well as the mean free time $\tau_{D}$. The intersection of the curves gives the number $n_{\max } \simeq 250$, and we conclude that we can ignore droplets with more than 250 enclosures on the time scale in question. Even so, the number of variables is by far too large for numerical computations and must be reduced.

\section{Reduction of variables}

\subsection{Number of enclosures}

In the present problem of coagulation, we have to consider the variables $N_{n}(V)$. A numerical solution requires discrete values $V_{A}$ for the volumes, and the number of variables $N_{n}^{A}$ might be extremely large, even if the maximum number of enclosures were small. Therefore, it is essential to reduce the number of variables.

To this objective it is reasonable to subsume droplets with adjacent numbers of enclosures into classes. These classes must not be of the same size. Indeed, it makes a big difference, whether a droplet contains 10 or 20 enclosures, while nobody would differentiate between two droplets with 10000 and 10010 enclosures. We shall use classes which contain an increasing number of enclosures.

We indicate the classes by Greek indices, $\alpha=1,2, \ldots, \alpha_{\max }$, and denote the number of members in class $\alpha$ by $\mu_{\alpha}$. Moreover, we define the boundaries of the classes by

$$
M_{\alpha}=\sum_{\beta=1}^{\alpha} \mu_{\beta} \quad \text { for } \alpha=1,2, \ldots, \alpha_{\max }, \quad \text { with } M_{0}=0 .
$$

There are no limitations for the choice of the class widths, $\mu_{\alpha}$. It turns out, however, that the doubling of the number of members of successive classes allows for simplifications of the subsequent calculations, and thus we set

$$
\mu_{\alpha}=2^{\alpha-2} \quad \text { for } \alpha=2, \ldots, \alpha_{\max } \quad \text { so that } M_{\alpha}=2^{\alpha-1} \text { for } \quad \alpha=1, \ldots, \alpha_{\max } .
$$


Obviously, we must have $M_{\alpha_{\max }}=n_{\max }$ and with the maximal number of enclosures given by $n_{\max } \simeq 250$, we have $\alpha_{\max }=9$.

With these definitions, we have for the number densities of droplets in class $\alpha$

$$
\nu_{\alpha}(V)=\sum_{n=M_{\alpha-1}+1}^{M_{\alpha}} N_{n}(V) \quad \text { for } \alpha=1,2, \ldots, \alpha_{\max } .
$$

Correspondingly, if the number density lies in class $\alpha$ it will be replaced by the mean number density, that is,

$$
N_{n}(V)=\frac{\nu_{\alpha}(V)}{\mu_{a}} \quad, \quad n \in\left\{M_{\alpha-1}+1, \ldots, M_{\alpha}\right\}
$$

The evolution equation for $\nu_{\alpha}(V)$ follows from summation of $(3)$, and after some rearrangements we obtain

$$
\begin{aligned}
& \frac{d \nu_{\alpha}(V)}{d t}=I_{\alpha-1, \alpha-1}(V)+\sum_{\beta=1}^{\alpha-1}\left(1-\mathcal{B}_{\alpha \beta}\right)\left[I_{\alpha \beta}(V)+I_{\beta \alpha}(V)\right] \\
& \\
&+\sum_{\beta=1}^{\alpha-2} \mathcal{B}_{\alpha-1, \beta}\left[I_{\alpha-1, \beta}(V)+I_{\beta, \alpha-1}(V)\right] \\
&-\sum_{\beta=1}^{\alpha_{\max }} \nu_{\alpha}(V) \int_{0}^{\infty} \sigma(V, U) \nu_{\beta}(U) d U+K_{0} \sum_{\beta=1}^{\alpha_{\max }} \mathcal{A}_{\alpha \beta} \frac{\nu_{\beta}(V)}{V},
\end{aligned}
$$

where we have abbreviated

$$
I_{\alpha \beta}(V)=\frac{1}{2} \int_{0}^{V} \sigma(U, V-U) \nu_{\alpha}(U) \nu_{\beta}(V-U) d U \quad \text { with } \quad I_{00}(V)=0 .
$$

The matrix $\mathcal{A}_{\alpha \beta}$ is given by sums of elements of the matrix $\mathcal{A}_{n m}$,

$$
\mathcal{A}_{\alpha \beta}=\begin{array}{ll}
\frac{1}{\mu_{\beta}} \sum_{n=1+M_{\alpha-1}}^{M_{\alpha}} \sum_{m=1+M_{\beta-1}}^{M_{\beta}} \frac{n m}{m-n}, & \beta>\alpha, \\
-\frac{1}{\mu_{\alpha}} \sum_{n=1+M_{\alpha-1}}^{M_{\alpha}} \sum_{m=1}^{M_{\alpha-1}} \frac{n m}{n-m}, & \beta=\alpha, \\
0, & \beta<\alpha,
\end{array}
$$

where $\omega_{\alpha}=\frac{1}{\mu_{\alpha}} \sum_{n=1+M_{\alpha-1}}^{M_{\alpha}} \sum_{m=1}^{M_{\alpha-1}} \frac{n m}{n-m}$ is the decay time for the class $\alpha$. Due to the large numbers involved, the elements of $\mathcal{A}_{\alpha \beta}$ for large values, that is, $\alpha, \beta \gtrsim 10$, should be computed by numerical integration rather than summation. The conservation of droplet number for non-colliding droplets follows from

$$
\sum_{\alpha=1}^{\alpha_{\max }} \mathcal{A}_{\alpha \beta}=\sum_{\alpha=1}^{\beta} \mathcal{A}_{\alpha \beta}=0
$$


for $\alpha_{\max } \geq \beta$, and, of course, this must be guaranteed also by numerical approximations to $\mathcal{A}_{\alpha \beta}$. The coefficients $\mathcal{B}_{\alpha \beta}$ are given by

$$
\mathcal{B}_{\alpha \beta}=\frac{\frac{1}{\mu_{\alpha}},}{\frac{3 \mu_{\beta}+1}{2 \mu_{\alpha}},}, \quad \beta \geq 2 .
$$

The relevant calculations, in particular the determination of $\mathcal{A}_{\alpha \beta}$ and $\mathcal{B}_{\alpha \beta}$, are outlined in Appendices A and B.

It is instructive to interpret the various contributions to the right hand side of (14). The first two lines refer to the gain of droplets of volume $V$ in class $\alpha$. The terms $I_{\alpha \beta}(V)$ refer to collisions of two droplets with number of enclosures in classes $\alpha$ and $\beta$ that produce a droplet of type $(V, \alpha)$. Note that due to the specific choice of the class width (12) a droplet in $\alpha$ will result only if one of the original droplets is in class $\alpha$ or $(\alpha-1)$, respectively.

The next terms refer to the loss of droplets $(V, \alpha)$ due to collisions with any other type of droplets, and to the gain and loss due to agglomeration of enclosures - the off-diagonal elements of $\mathcal{A}_{\alpha \beta}$ refer to the gain and the diagonal terms to the loss.

Of course, the total number density of droplets with volume $V$ is given by

$$
\mathcal{N}(V)=\sum_{\alpha=1}^{\alpha_{\max }} \nu_{a}(V)
$$

The summation of (14) over all classes should give the coagulation equation for droplets (2). It is easy to show that this is indeed the case as long as $\alpha_{\max } \rightarrow \infty$ or as $\nu_{\alpha}(V) \simeq 0$ for $\alpha \geq \alpha_{\max }$, respectively. The last condition gives us a useful criterion for the choice of $\alpha_{\max }$ : if during the numerical calculation $\nu_{a_{\max }}$ becomes significantly different from zero, the number $\alpha_{\max }$ is too small for the process under consideration, and must be increased.

\subsection{Volume of droplets}

In the last step for the reduction of variables, we subsume the continuous volumes of the droplets into sections, see [10] for a thorough survey for the case without enclosures. We indicate the sections by capital indices, $A=1,2, \ldots, A_{\max }$, and denote the volumes of the sections by $\Delta_{A}$. Moreover, we define the boundaries of the sections by

$$
V_{A}=\sum_{B=1}^{A} \Delta_{B} \quad \text { for } A=1,2, \ldots, A_{\max } \quad \text { with } V_{0}=0 .
$$

Again, the doubling of the volumes of successive sections allows for simplifications of the subsequent calculations, and thus we set

$$
\Delta_{A}=2^{A-2} \bar{V} \quad \text { for } A=2, \ldots, A_{\max } \quad \text { so that } V_{A}=2^{A-1} \bar{V} \quad \text { for } A=1, \ldots, A_{\max } .
$$


Here, $\bar{V}$ defines the smallest droplet volume of interest and must be chosen according to the process under consideration.

With these definitions, we have for the number densities of droplets with number of enclosures in class $\alpha$ and volume in section $A$

$$
\nu_{\alpha}^{A}=\int_{V_{A-1}}^{V_{A}} \nu_{\alpha}(V) d V \quad, \quad A=1,2, \ldots, A_{\max } .
$$

Correspondingly, if the number density lies in class $\alpha$ and section $A$, it will be replaced by the mean number density

$$
\nu_{\alpha}(V)=\frac{\nu_{\alpha}^{A}}{\Delta_{A}} \quad, \quad V \in\left(V_{A-1}, V_{A}\right) .
$$

The evolution equation for the number densities $\nu_{\alpha}^{A}$ follows from integration of (14) over the interval $\left(V_{A-1}, V_{A}\right)$ and reads after some rearrangements

$$
\begin{aligned}
\frac{d \nu_{\alpha}^{A}}{d t}=I_{\alpha-1, \alpha-1}^{A} & +\sum_{\beta=1}^{\alpha-1}\left(1-\mathcal{B}_{\alpha \beta}\right)\left[I_{\alpha \beta}^{A}+I_{\beta \alpha}^{A}\right] \\
& +\sum_{\beta=1}^{\alpha-2} \mathcal{B}_{\alpha-1, \beta}\left[I_{\alpha-1, \beta}^{A}+I_{\beta, \alpha-1}^{A}\right] \\
& -\sum_{\beta=1}^{\alpha_{\max }} \sum_{B=1}^{A_{\max }} \sigma_{A B} \nu_{\alpha}^{A} \nu_{\beta}^{B}+\frac{K_{0} \ln 2}{\Delta_{A}} \sum_{\beta=\alpha}^{\alpha_{\max }} \mathcal{A}_{\alpha \beta} \nu_{\beta}^{A} .
\end{aligned}
$$

Some details of the calculation are given in Appendix C. In particular, $I_{\alpha \beta}^{A}$ is an abbreviation for a non-linear function of the variables $\nu_{\alpha}^{A}$ given by

$$
\begin{aligned}
I_{00}^{A}= & 0 \\
I_{\alpha \beta}^{1}= & \frac{1}{2}\left(\sigma_{11}-\hat{\sigma}_{11}\right) \nu_{\alpha}^{1} \nu_{\beta}^{1}, \\
I_{\alpha \beta}^{2}= & \frac{1}{2} \hat{\sigma}_{11} \nu_{\alpha}^{1} \nu_{\beta}^{1}+\frac{1}{2}\left(\sigma_{21}-\hat{\sigma}_{21}\right)\left[\nu_{\alpha}^{1} \nu_{\beta}^{2}+\nu_{\alpha}^{2} \nu_{\beta}^{1}\right], \\
I_{\alpha \beta}^{A}=\frac{1}{2}\left[\sigma_{A-1, A-1} \nu_{\alpha}^{A-1} \nu_{\beta}^{A-1}+\sum_{B=1}^{A-2} \hat{\sigma}_{A-1, B}\left[\nu_{\alpha}^{A-1} \nu_{\beta}^{B}+\nu_{\alpha}^{B} \nu_{\beta}^{A-1}\right]\right. & \\
& \left.+\sum_{B=1}^{A-1}\left(\sigma_{A B}-\hat{\sigma}_{A B}\right)\left[\nu_{\alpha}^{A} \nu_{\beta}^{B}+\nu_{\alpha}^{B} \nu_{\beta}^{A}\right]\right] \quad \text { for } A \geq 3
\end{aligned}
$$

where $\sigma_{A B}$ and $\hat{\sigma}_{A B}$ are mean collision frequencies for the droplets and are computed by

$$
\sigma_{A B}=\int_{V_{B-1}}^{V_{B}} \int_{V_{A-1}}^{V_{A}} \frac{\sigma(U, W)}{\Delta_{A} \Delta_{B}} d W d U \quad, \quad \hat{\sigma}_{A B}=\int_{V_{B-1}}^{V_{B}} \int_{V_{A}-U}^{V_{A}} \frac{\sigma(U, W)}{\Delta_{A} \Delta_{B}} d W d U .
$$


The interpretation of the various terms is as follows: terms in $I_{\alpha \beta}^{A}$ with $\sigma_{A B}, \hat{\sigma}_{A B}$ and $\sigma_{A-1, B}$, $\hat{\sigma}_{A-1, B}$ refer to the gain of droplets in section $A$ due to collision of droplets in section $A$ and $B$ or the collision of droplets in $(A-1)$ and $B$, respectively. Again, the terms in the last line of (22) refer to the loss due to collisions of droplets of type $(\alpha, A)$ with any other type $(\beta, B)$ and to the coagulation of enclosures, described by the matrix $\mathcal{A}_{\alpha \beta}$.

The original equation (3) conserves the total volume density, $v_{\text {tot }}(t)$, as defined in (4). In terms of the discrete variables $\nu_{\alpha}^{A}$, we can define the total volume as

$$
v_{\text {tot }}(t)=\sum_{A} \bar{V}_{A} \sum_{\alpha} \nu_{\alpha}^{A}
$$

where $\bar{V}_{A}$ is a suitable mean droplet volume associated with Section $A$. We have not checked the conservation analytically, but rely on the numerical results below. It turns out that the volume is well conserved (error less than 1\%) if one chooses

$$
\bar{V}_{A}=\frac{\left(\int_{V_{a-1}}^{V_{A}} V^{a} d V\right)^{\frac{1}{a}}}{\int_{V_{a-1}}^{V_{A}} V^{a} d V} \text { with } a \simeq 1.16
$$

where the value of the exponent $a$ was found empirically.

\subsection{Dimensionless quantities, time scales}

In this section, we introduce dimensionless quantities in order to identify the relevant parameters and timescales of our problem. It is natural to measure the volume in multiples of the volume $\bar{V}$, which is the smallest possible droplet volume. Its value is determined by the initial conditions.

With $u, w$ defined as $U=u \bar{V}, W=w \bar{V}$, we can write the collision probability as

$$
\sigma(U, W)=\sigma_{0} \sigma(u, w)
$$

where

$$
\sigma_{0}=\left(\frac{3}{4 \pi}\right)^{1 / 6}\left(\frac{6 k T}{\varrho \bar{V}}\right)^{1 / 2} \bar{V}^{2 / 3} \text { and } \sigma(u, w)=\left(\frac{1}{u}+\frac{1}{w}\right)^{1 / 2}\left(v^{1 / 3}+w^{1 / 3}\right)^{2}
$$

The dimensionless time is best defined as

$$
\tilde{t}=\frac{\sigma_{0}}{\bar{V}} t
$$

and the parameter

$$
\tilde{K}_{0}=\frac{K_{0}}{\sigma_{0}}
$$


measures the relative frequency of the coagulation of enclosures. Moreover, we introduce the dimensionless section widths by

$$
\delta_{A}=\Delta_{A} / \bar{V} \quad \text { and } \quad v_{A}=V_{A} / \bar{V}=\sum_{B=1}^{A} \delta_{B}
$$

and replace $\nu_{\alpha}^{A}$ by the dimensionless expression

$$
\tilde{\nu}_{\alpha}^{A}=\nu_{\alpha}^{A} \bar{V}
$$

For simplicity of the notation, we omit the tildes in the notation, so that the equation in dimensionless units reads

$$
\begin{gathered}
\frac{d \nu_{\alpha}^{A}}{d t}=I_{\alpha-1, \alpha-1}^{A}+\sum_{\beta=1}^{\alpha-1}\left(1-\mathcal{B}_{\alpha \beta}\right)\left[I_{\alpha \beta}^{A}+I_{\beta \alpha}^{A}\right]+\sum_{\beta=1}^{\alpha-2} \mathcal{B}_{\alpha-1, \beta}\left[I_{\alpha-1, \beta}^{A}+I_{\beta, \alpha-1}^{A}\right] \\
-\sum_{\beta=1}^{\alpha_{\max }} \sum_{B=1}^{A_{\max }} \sigma_{A B} \nu_{\alpha}^{A} \nu_{\beta}^{B}+K_{0} \frac{\ln 2}{\delta_{A}} \sum_{\beta=\alpha}^{\alpha_{\max }} \mathcal{A}_{\alpha \beta} \nu_{\beta}^{A} .
\end{gathered}
$$

The dimensionless counterpart of $I_{\alpha \beta}^{A}$ has exactly the same form as $I_{\alpha \beta}^{A}(23)$, where now $\sigma_{A B}, \hat{\sigma}_{A B}$ stands for the dimensionless quantities.

$$
\sigma_{A B}=\int_{v_{A-1}}^{v_{A}} \int_{v_{B-1}}^{v_{B}} \frac{\sigma(u, w)}{\delta_{A} \delta_{B}} d w d u \quad, \quad \hat{\sigma}_{A B}=\int_{v_{A-1}}^{v_{A}} \int_{v_{B-1}-w}^{v_{B}} \frac{\sigma(u, w)}{\delta_{A} \delta_{B}} d w d u .
$$

The matrices $\mathcal{A}_{\alpha \beta}, \mathcal{B}_{\alpha \beta}$ are numbers, and remain unchanged.

\section{$5 \quad$ Results}

We consider a case where initially all droplets have the same volume $\bar{V}=\frac{4}{3} \pi \bar{r}^{3}$ with a radius $\bar{r}=510^{-9} \mathrm{~m}$; the initial number density of droplets is $\mathcal{N}_{0}(\bar{V})=10^{18} \mathrm{~m}^{-3}$. For the calculation of $K_{0}$, we need the viscosity of silica. Extrapolating data from [9](page 764), we find

$$
\eta=10^{-8.6625\left(1-\frac{3556.03 K}{T}\right)} \frac{k g}{m s} .
$$

With this formula, we have for the dimensionless values of $K_{0}$ at $T=2300 \mathrm{~K}$ and $T=2600 \mathrm{~K}$

$$
K_{0}(2300 \mathrm{~K})=1.1410^{-9}, \quad K_{0}(2600 \mathrm{~K})=4.2510^{-8} .
$$

The initial number of enclosures in the droplets is assumed to be the maximum number we can observe on the time scale defined by the collisions. By the same arguments as in Section 3.3 , we obtain for $T=2300 \mathrm{~K}$ an initial number of $n_{\max } \simeq 30$, corresponding to the class 
$\alpha_{\max }=6$ and for $T=2600 \mathrm{~K}$ we find $\alpha_{\max }=3$. The corresponding initial condition is given by

$$
\nu_{\alpha}^{A}(t=0)=u_{0} \delta_{A, 1} \delta_{\alpha, \alpha_{\max }}, \quad u_{0}=\bar{V} \mathcal{N}_{0}(\bar{V}) \simeq 510^{-7}
$$

We compute the coagulation process for $T=2300 \mathrm{~K}$, accounting for 12 classes and 15 sections, and for $T=2600 \mathrm{~K}$ accounting for 9 classes and 15 sections, respectively.

The contour plots in Figure 5 show, for $T=2300 \mathrm{~K}$, the temporal development of the aerosol through $\nu_{\alpha}^{A}(A$ : $x$-axis, $\alpha$ : $y$-axis). Recall that $A$ is a measure of the drop size and $\alpha$ is a measure of the number of enclosures. We observe the development of a structure which moves in the $(A, \alpha)$-plane almost without changing its shape. The development of a self-preserving structure is well known for the coagulation process of droplets alone [7]. Here, we observe a similar behavior for droplets with enclosures.

The diagonal shape of the structure becomes clear if one thinks of the relevant growth mechanisms: Droplets with a large volume and a large number of enclosures are those that were newly produced by collisions of smaller droplets with less enclosures. Small droplets vanish due to collisions. And finally, the number of droplets with a large number of enclosures decreases due to enclosure coagulation. Small droplets are those that have encountered fewer collisions, and therefore, their number of enclosures - never increased by collisions, but rather decreased by coagulation - is at the bottom left hand region of the diagonal structure.

Figure 6 shows the coagulation process with the same parameters, but for a case where initially all droplets have only one enclosure. At early times, the contour plots differ from those in Figure 5, but with increasing time, we find the same self-preserving structure as before - the result becomes independent of the initial condition.

If one chooses the initial number of enclosures to be larger than $n_{\max }$, one observes first a decrease of the number of enclosures, with no coagulation of droplets, corresponding to the results in Section 3.2. This reflects the difference in the characteristic time scales for the two coagulation processes. When the number of enclosures has decreased to $n_{\max }$ as computed in Section 3.3, the characteristic times become comparable, the coagulation of droplets sets in, and the results are close to those in Figure 5.

In Figure 7 we show the result for $T=2600 \mathrm{~K}$. Due to the higher temperature, the enclosures move more easily and thus coagulate more frequent. Therefore, the self preserving structure is located at lower numbers of enclosures.

In all cases, the structure travels almost horizontally, with a slight ascent towards the right upper corner. Thus, in later times, the number of enclosures is increasing. In order to make this behavior more evident, Figure 8 shows the mean volume of enclosures

$$
\bar{v}=\sum_{\alpha, A} \frac{c \bar{V}_{A}}{\bar{n}_{\alpha}} \nu_{\alpha}^{A} / \sum_{\alpha, A} \nu_{\alpha}^{A}
$$


and the mean number of enclosures

$$
\bar{n}=\sum_{\alpha, A} \bar{n}_{\alpha} \nu_{\alpha}^{A} / \sum_{\alpha, A} \nu_{\alpha}^{A}
$$

where $\bar{V}_{A}$ is the mean volume of droplets in section $A$, see $(25)$, and $\bar{n}_{\alpha}=\frac{2^{\alpha-1}+2^{\alpha-2}}{2}$ is the mean number of enclosures in class $\alpha$. The mean volume was calculated for concentration $c=0.1$.

Both mean volume and mean number of enclosures increase in time. This is in contradiction to the experimental findings which show a decrease in the number of enclosures at later times, see Figure 1, 2. Mathematically, the ascent is related to the term $K_{0} / V$ in the collision probability for enclosures, $\gamma_{m \rightarrow n}(6)$, which means that in larger droplets the enclosures coagulate less frequently. We have to conclude that our model for $\gamma_{m \rightarrow n}$ is to simple. Indeed, in the derivation of $\gamma_{m \rightarrow n}$, we have ignored the size distribution of the enclosures, as well as the finite size of the droplets, in particular the tendency of the enclosures to stay at the edge of the droplets (see Figure 1).

Clearly, we would like to see an increase in the enclosure collision rate, which could occur if the volume dependence in (6) was smaller. One justification would be that as we noted experimental evidence suggests a migration of the enclosures to the surface, implying a surface diffusion of enclosures, or alternatively a decrease in the effective volume used in the denominator of (6). This argument would favor an exponent of 0.66 for $V$. We found that if we replace $\gamma_{m \rightarrow n}$ by

$$
\tilde{\gamma}_{m \rightarrow n}(V)=\frac{K_{0}}{V^{1 / 3}} \frac{n m}{m-n}
$$

we can find the desired behavior. The choice of the exponent $(1 / 3)$ is arbitrary and not justified by any physical considerations. Figure 9 shows the result. Now, indeed, the number of enclosures decreases with increasing time.

The enhancement of the collision rate is not unjustified if we consider the surface effects discussed above as well as the fact that our present model assume monodisperse enclosures. This results show that a better modeling of the inner droplet processes as well as the incorporation of a poly-disperse enclosure model might allow for better agreement with the experiments.

\section{A The matrix $\mathcal{A}_{\alpha \beta}$}

For the determination of the matrix $\mathcal{A}_{\alpha \beta}$, we consider

$$
S_{\alpha}=\sum_{n=M_{\alpha-1}+1}^{M_{\alpha}}\left[\sum_{m=n+1}^{n_{\max }} \frac{n m}{m-n} N_{m}-\sum_{m=1}^{n-1} \frac{n m}{n-m} N_{n}\right] \text {. }
$$


With (13) and $n_{\max }=M_{\alpha_{\max }}$, we can write

$$
\begin{aligned}
S_{\alpha} & =\sum_{n=M_{\alpha-1}+1}^{M_{\alpha}}\left[\sum_{m=n+1}^{M_{\alpha}} \frac{n m}{m-n} N_{m}+\sum_{\beta=\alpha+1}^{\alpha_{\max }} \sum_{m=M_{\beta-1}+1}^{M_{\beta}} \frac{n m}{m-n} N_{m}-\sum_{m=1}^{n-1} \frac{n m}{n-m} N_{n}\right] \\
& =\sum_{\beta=\alpha+1}^{\alpha_{\max }} \frac{1}{\mu_{\beta}} \sum_{n=M_{\alpha-1}+1}^{M_{\alpha}} \sum_{m=M_{\beta-1}+1}^{M_{\beta}} \frac{n m}{m-n} \nu_{\beta}-\frac{1}{\mu_{\alpha}} \sum_{n=M_{\alpha-1}+1}^{M_{\alpha}}\left[\sum_{m=n+1}^{M_{\alpha}} \frac{n m}{n-m}+\sum_{m=1}^{n-1} \frac{n m}{n-m}\right] \nu_{\alpha} .
\end{aligned}
$$

For the second expression, we have

$$
\sum_{n=M_{\alpha-1}+1}^{M_{\alpha}}\left[\sum_{m=n+1}^{M_{\alpha}} \frac{n m}{n-m}+\sum_{m=1}^{n-1} \frac{n m}{n-m}\right]=\sum_{n=M_{\alpha-1}+1}^{M_{\alpha}} \sum_{\substack{m=1 \\ m \neq n}}^{M_{\alpha}} \frac{n m}{n-m}=\sum_{n=M_{\alpha-1}+1}^{M_{\alpha}} \sum_{m=1}^{M_{\alpha-1}} \frac{n m}{n-m}
$$

where we have used that, due to symmetry,

$$
\sum_{n=M_{\alpha-1}+1}^{M_{\alpha}} \sum_{\substack{m=M_{\alpha-1}+1 \\ m \neq n}}^{M_{\alpha}} \frac{n m}{n-m}=0
$$

We obtain finally that

$$
S_{\alpha}=\sum_{\beta=\alpha+1}^{\alpha_{\max }} \mathcal{A}_{\alpha \beta} \nu_{\beta}+\mathcal{A}_{\alpha \alpha} \nu_{\alpha}
$$

with the matrix elements given by (16).

\section{B The matrix $\mathcal{B}_{\alpha \beta}$}

We write $N_{m}(U)=N_{m}, N_{m}(U-V)=\bar{N}_{m}$ and consider the quantity

$$
S_{\alpha}=\sum_{n=M_{\alpha-1}+1}^{M_{\alpha}} \sum_{m=1}^{n-1} N_{m} \bar{N}_{n-m}
$$

that appears in the summation of the first term on the right hand side of (3) over the class $\alpha$. This can be written as

$$
S_{\alpha}=s_{\alpha}-s_{\alpha-1}
$$

where

$$
s_{\alpha}=\sum_{n=2}^{M_{\alpha}} \sum_{m=1}^{n-1} N_{m} \bar{N}_{n-m}=\sum_{m=1}^{M_{\alpha}-1} \sum_{n=m+1}^{M_{\alpha}} N_{m} \bar{N}_{n-m}=\sum_{m=1}^{M_{\alpha}-1} \sum_{n=1}^{M_{\alpha}-m} N_{m} \bar{N}_{n} \text { and } s_{0}=s_{1}=0 .
$$


The reversion of the order of summation is best understood by means of Figure 10, that shows the area of summation for $s_{\alpha}$. It involves also the substitution $(n-m) \rightarrow n$. The meaning of $S_{\alpha}$ is best read off from Figure 11 which pictures the situation for the calculation of $S_{6}$ :

$s_{6}$ is the sum over all points on and below the upper dashed diagonal, and $s_{5}$ is the sum over all points below (but not on) the lower dashed diagonal.

$S_{6}$ is the sum over all those points on and inside the dashed curve in the figure.

All points inside the rectangles $\left(1+M_{\beta-1}, M_{\beta}\right)\left(1+M_{\gamma-1}, M_{\gamma}\right)$ refer to values of $N_{m} \bar{N}_{n}=$ $\nu_{\beta} \bar{\nu}_{\gamma} /\left(\mu_{\beta} \mu_{\gamma}\right)$, see Eq. (13). For the calculation of $S_{\alpha}$ we have to multiply $\nu_{\beta} \bar{\nu}_{\gamma} /\left(\mu_{\beta} \mu_{\gamma}\right)$ with the number of those points of the rectangles that lie inside the dashed region, and sum over all rectangles. It is due to our peculiar choice (12) of the class width $\mu_{\alpha}$ that $S_{\alpha}$ can be calculated easily as

$$
\begin{aligned}
S_{\alpha} & =\nu_{\alpha-1} \bar{\nu}_{a-1}+\sum_{\beta=2}^{\alpha-2} \frac{3 \mu_{\beta}^{2}}{2 \mu_{\alpha-1} \mu_{\beta}}\left(\nu_{\alpha-1} \bar{\nu}_{\beta}+\nu_{\beta} \bar{\nu}_{\alpha-1}\right)+\frac{1}{\mu_{\alpha-1}}\left(\nu_{\alpha-1} \bar{\nu}_{1}+\nu_{1} \bar{\nu}_{\alpha-1}\right) \\
& +\sum_{\beta=2}^{\alpha-1} \frac{\mu_{\alpha} \mu_{\beta}-\frac{3}{2} \mu_{\beta}^{2}}{\mu_{\alpha} \mu_{\beta}}\left(\nu_{\alpha} \bar{\nu}_{\beta}+\nu_{\beta} \bar{\nu}_{\alpha}\right)+\frac{\mu_{\alpha}-1}{\mu_{\alpha}}\left(\nu_{\alpha} \bar{\nu}_{1}+\nu_{1} \bar{\nu}_{\alpha}\right) .
\end{aligned}
$$

Introducing the matrix $\mathcal{B}_{\alpha \beta}$ given by (10), we find finally

$$
\begin{aligned}
S_{1} & =0 \\
S_{\alpha} & =\nu_{\alpha-1} \bar{\nu}_{a-1}+\sum_{\beta=1}^{\alpha-1}\left(1-\mathcal{B}_{\alpha \beta}\right)\left(\nu_{\alpha} \bar{\nu}_{\beta}+\nu_{\beta} \bar{\nu}_{\alpha}\right)+\sum_{\beta=1}^{\alpha-2} \mathcal{B}_{\alpha-1, \beta}\left(\nu_{\alpha-1} \bar{\nu}_{\beta}+\nu_{\beta} \bar{\nu}_{\alpha-1}\right) .
\end{aligned}
$$

Note that $\mathcal{B}_{21}=\frac{1}{\mu_{2}}=1$, so that $S_{2}=\nu_{1} \bar{\nu}_{1}$.

\section{The functions $I_{\alpha \beta}^{A}$}

We compute

$$
I_{\alpha \beta}^{A}=\int_{V_{A-1}}^{V_{A}} I_{\alpha \beta}(V) d V=\frac{1}{2} \int_{V_{A-1}}^{V_{A}} \int_{0}^{V} \sigma(U, V-U) \nu_{\alpha}(U) \nu_{\beta}(V-U) d U d V .
$$

which after the substitution $V-U \rightarrow W$ can be written as

$$
I_{\alpha \beta}^{A}=i_{\alpha \beta}^{A}-i_{\alpha \beta}^{A-1} \quad \text { with } \quad i_{\alpha \beta}^{A}=\frac{1}{2} \int_{0}^{V_{A}} \int_{0}^{V_{A}-U} \sigma(U, W) \nu_{\alpha}(U) \nu_{\beta}(W) d W d U, i_{\alpha \beta}^{0}=0 .
$$

The meaning of $I_{\alpha \beta}^{A}$ is best read off from Fig. 12 which shows the situation for the calculation of $I_{\alpha \beta}^{6}$ : All points inside the rectangles $\left(V_{A-1}, V_{A}\right)\left(V_{B-1}, V_{B}\right)$ refer to values of 
$\nu_{\alpha}(U) \nu_{\beta}(W)=\nu_{\alpha}^{A} \nu_{\beta}^{B} /\left(\Delta_{A} \Delta_{B}\right)$, see (21). $I_{6}$ is the integral over the dashed curve in the figure. After replacing the number densities in the sections with the mean values (21) we find

$$
\begin{gathered}
I_{\alpha \beta}^{1}=\frac{1}{2} \int_{0}^{V_{1}} \int_{0}^{V_{1}-U} \frac{\sigma(U, W)}{\Delta_{1} \Delta_{1}} d W d U \nu_{\alpha}^{1} \nu_{\beta}^{1}, \\
I_{\alpha \beta}^{2}=\frac{1}{2} \int_{0}^{V_{1}} \int_{V_{1}-U}^{V !} \frac{\sigma(U, W)}{\Delta_{1} \Delta_{1}} d W d U \nu_{\alpha}^{1} \nu_{\beta}^{1}+\frac{1}{2} \int_{0}^{V_{1}} \int_{V_{1}}^{V_{2}-U} \frac{\sigma(U, W)}{\Delta_{1} \Delta_{2}} d W d U\left[\nu_{\alpha}^{1} \nu_{\beta}^{2}+\nu_{\alpha}^{2} \nu_{\beta}^{1}\right], \\
I_{\alpha \beta}^{A}=\frac{1}{2} \int_{V_{A-2}}^{V_{A-1}} \int_{V_{A-2}}^{V_{A-1}} \frac{\sigma(U, W)}{\Delta_{A-1} \Delta_{A-1}} d W d U \nu_{\alpha}^{A-1} \nu_{\beta}^{A-1} \\
+\frac{1}{2} \sum_{B=1}^{A-2} \int_{V_{B-1}}^{V_{B}} \int_{V_{A-1}-U}^{V_{A-1}} \frac{\sigma(U, W)}{\Delta_{A-1} \Delta_{B}} d W d U\left[\nu_{\alpha}^{A-1} \nu_{\beta}^{B}+\nu_{\alpha}^{B} \nu_{\beta}^{A-1}\right] \\
+\frac{1}{2} \sum_{B=1}^{A-1} \int_{V_{B-1}}^{V_{B}} \int_{V_{A-1}}^{V_{A}-U} \frac{\sigma(U, W)}{\Delta_{A} \Delta_{B}} d W d U\left[\nu_{\alpha}^{A} \nu_{\beta}^{B}+\nu_{\alpha}^{B} \nu_{\beta}^{A}\right] \quad(A \geq 3) .
\end{gathered}
$$

Introduction of the mean collision frequencies (24) yields $I_{\alpha \beta}^{A}$ in the form (23).

\section{References}

[1] P. Biswas, C.Y. Wu , M.R. Zachariah, and B.K. McMillen, In Situ Characterization of Vapor Phase Growth of Iron Oxide-Silica Nanocomposite; Part II: Comparison of a Discrete- Sectional Model Predictions to Experimental Data. J. Mat. Res. 12, 714 (1997)]

[2] P. Biswas and M.R. Zachariah, In Situ Immobilization of Lead Species in Combustion Environments by Injection of Gas Phase Silica Sorbent Precursors. Env. Sci. Tech. 31, 2455 (1997)

[3] P. Biswas, G. Yang and M.R. Zachariah, In-Situ Processing of Ferroelectric Materials from Lead Streams by Injection of Gas-Phase Titanium Precursors. Combustion Science and Technology 134, 183 (1998)

[4] S.H. Ehrman, S.K. Friedlander and M.R. Zachariah. Characterization of $\mathrm{SiO}_{2} / \mathrm{TiO}_{2}$ Nanocomposite Aerosol in a Premixed Flame. J. Aerosol Science 29, 687 (1998)

[5] S.H. Ehrman, M.I. Aquino-Class and M.R. Zachariah, Effect of temperature and vaporphase encapsulation on particle growth and morphology. J. Materials Research 14(4), 1664-1671 (1999) 
[6] S.H. Ehrman, S.K. Friedlander and M.R. Zachariah, Phase Segregation in Binary $\mathrm{SiO}_{2} / \mathrm{TiO}_{2}, \mathrm{SiO}_{2} / \mathrm{Fe}_{2} \mathrm{O}_{3}$ Aerosols Formed in a Premixed Flame. J. Mater. Res. 14, 4551 (1999]

[7] S.K. Friedlander, Smoke, Dust and Haze, Oxford 2000

[8] N.A. Fuchs The mechanics of aerosols. Dover, New York 1989

[9] W.D. Kingery, H.K. Bowen and D.R. Uhlmann, Introduction to ceramics. Wiley, New York 1976

[10] J.D. Landgrebe and S.E. Pratsinis A discrete-sectional model for particulate production by gas-phase chemical reaction and aerosol coagulation in the free-molecular regime. J. Colloid Interface Sci. 139, 63-86 (1990)

[11] B. K. McMillin, P. Biswas and M.R. Zachariah, In Situ Characterization of Vapor Phase Growth of Iron Oxide-Silica Nanocomposite; Part I: 2-D Planar Laser-Induced Fluorescence and Mie Imaging. J. Mat. Res. 11, 1552-1561 (1996)

[12] S.H. Park, K.W. Lee, E. Otto and H. Fissan The log-normal size distribution theory of Brownian aerosol coagulation for the entire particle range: Part I: Analytical solution using the harmonic mean coagulation kernel. J. Aerosol. Sci 30, 3-16 (1999)

[13] M.R. Zachariah, M. Aquino-Class, R.D. Shull and E. Steel, Formation of Superparamagnetic Nanocomposites from Vapor Phase Condensation in a Flame. Nanostructured Materials 5, 383 (1995)

[14] M.R. Zachariah, R.D. Shull, B.K. McMillin and P. Biswas, In-Situ Characterization and Modeling of the Vapor Phase Growth of a Superparamagnetic Nanocomposite. Nanotechnology, Vol. 622, Chapter 3, Pg. 42-63 (1996) 


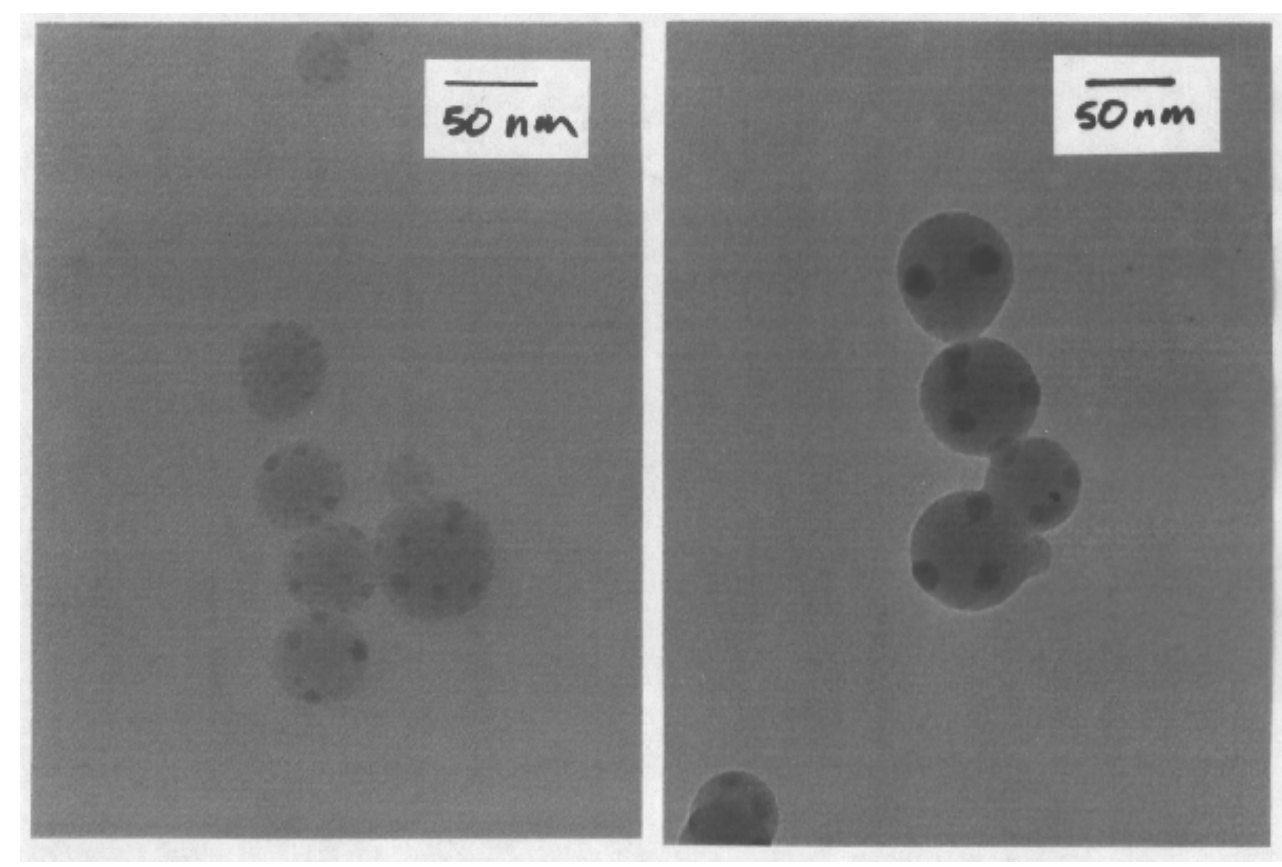

Figure 1: Aerosol droplet with enclosures. The droplets on the left are "younger".

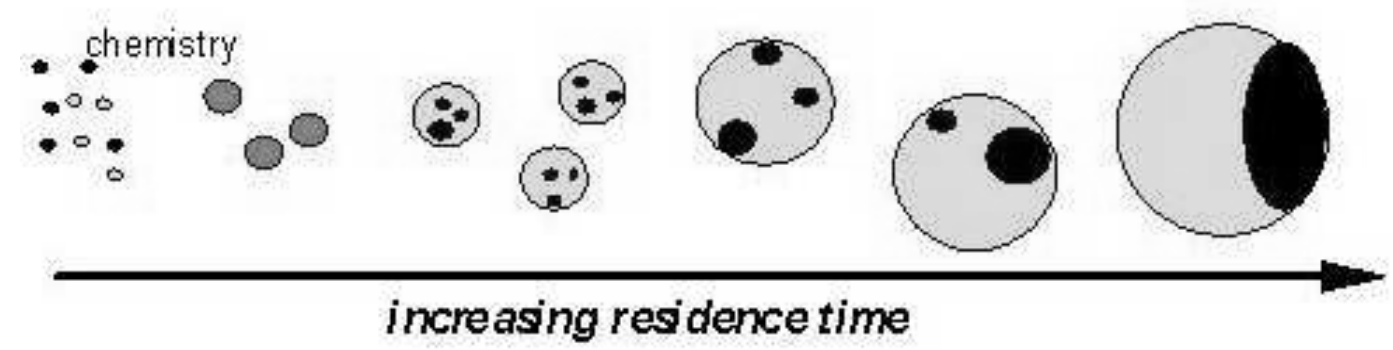

Figure 2: Schematic of the temporal evolution of a two component aerosol. 

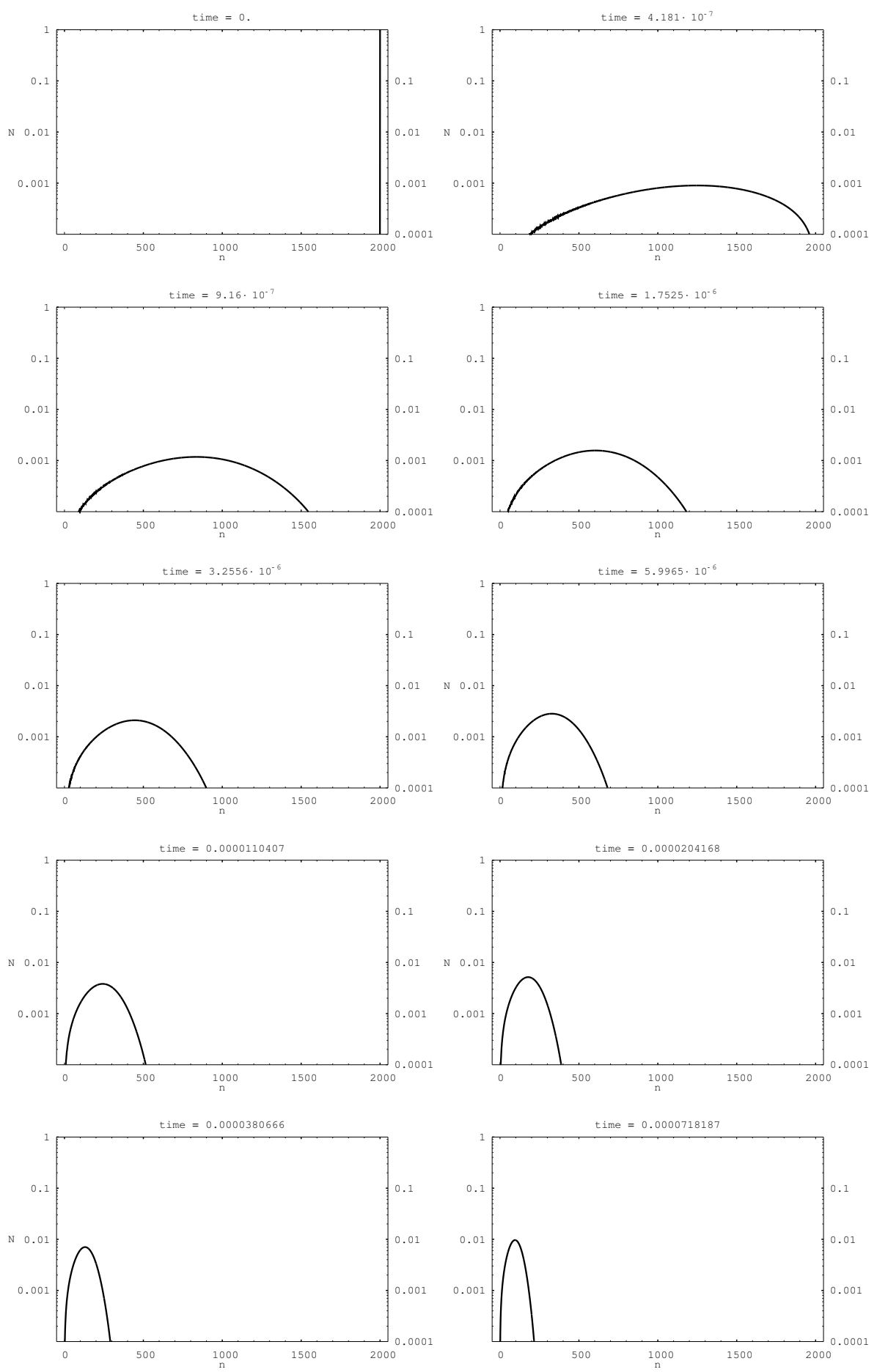

Figure 3: Distribution of droplets with $n$ enclosures for various times. $n_{\max }=2000$, initial conditions $N_{n_{\max }}(0)=1, N_{n}(0)=0$. 


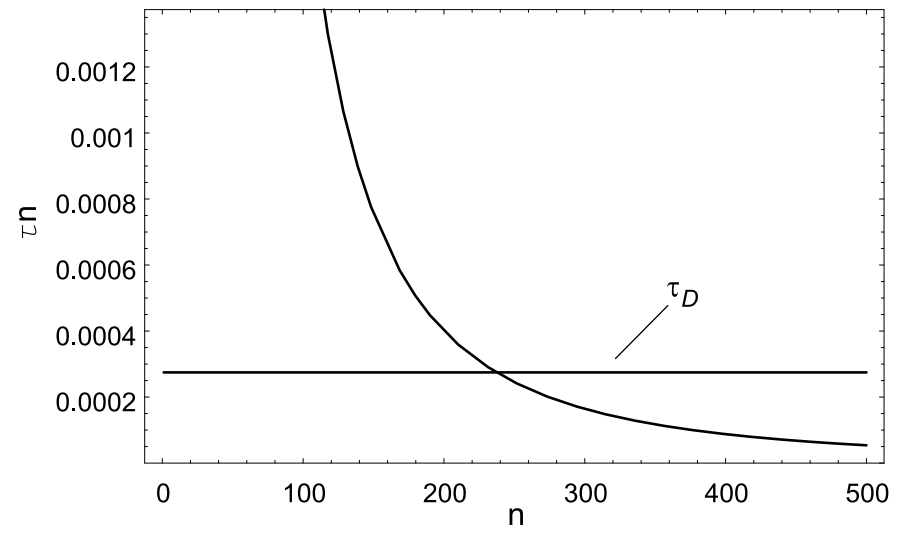

Figure 4: Characteristic times $\tau_{n}$ and $\tau_{D}$ for silica at $T=2000 K$ (in seconds). The intersection of the curves defines $n_{\max } \simeq 250$. 


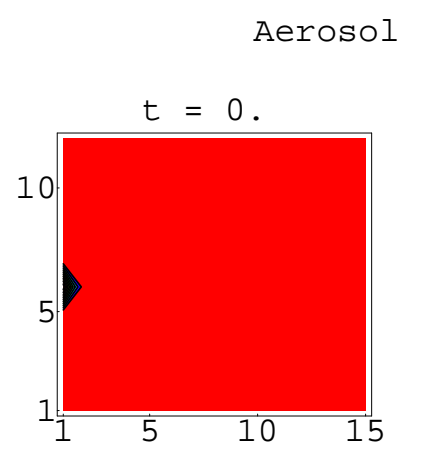

$\mathrm{K}=1.14 \cdot 10^{-9} \mathrm{u} 0=5 \cdot \cdot 10^{-7}$
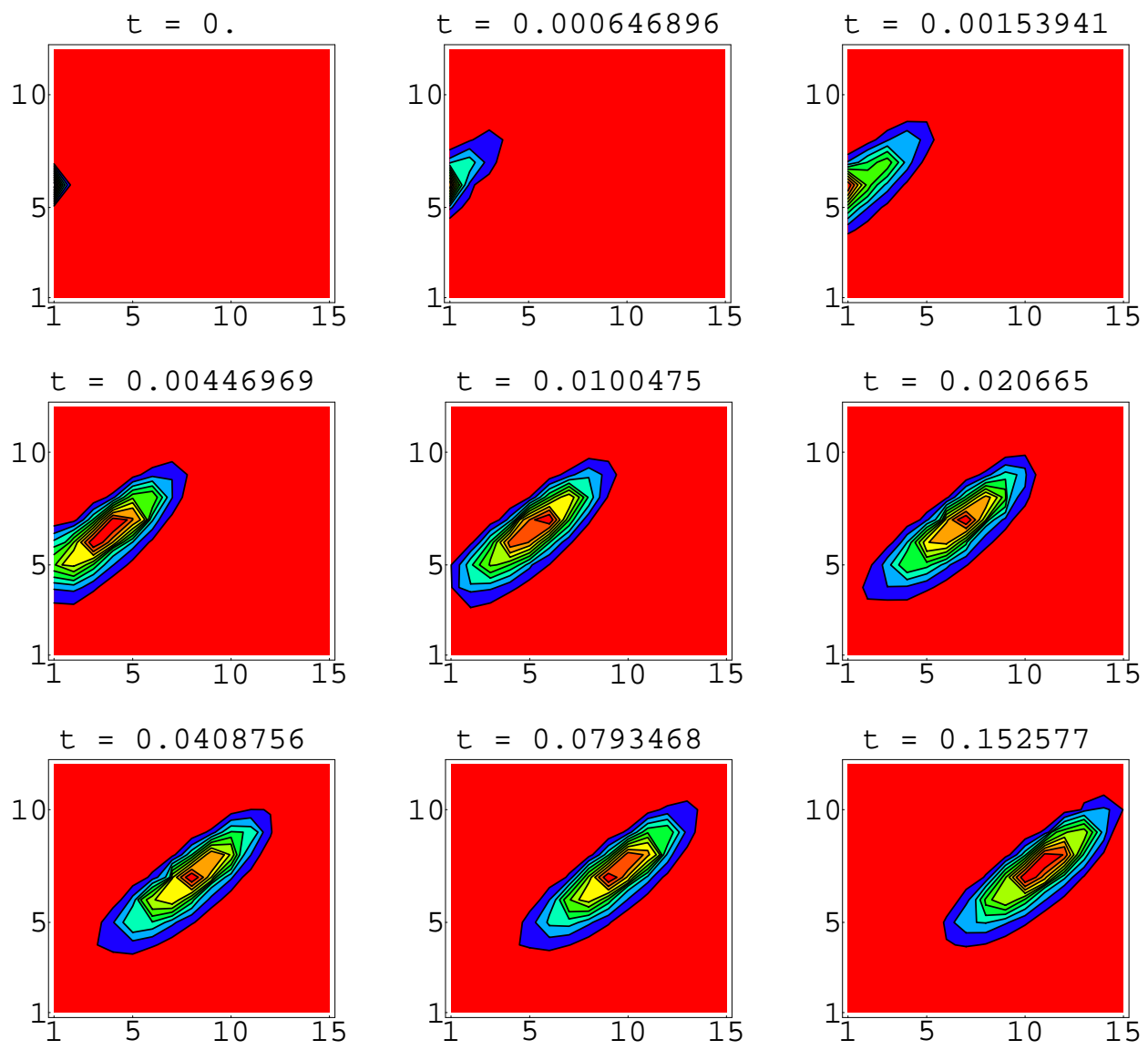

Figure 5: Temporal evolution of for $K=1.1410^{-9}, u_{0}=510^{-7}(T=2300 K)$. The gray levels refer only to the relative values inside one plot. $t$ is the actual time in seconds. 


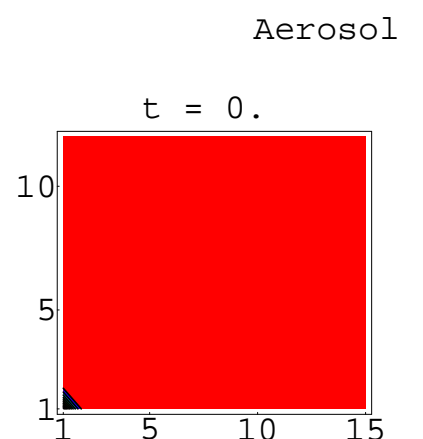

$\mathrm{K}=1.14 \cdot 10^{-9} \quad \mathrm{u} 0=5 \cdot \cdot 10^{-7}$
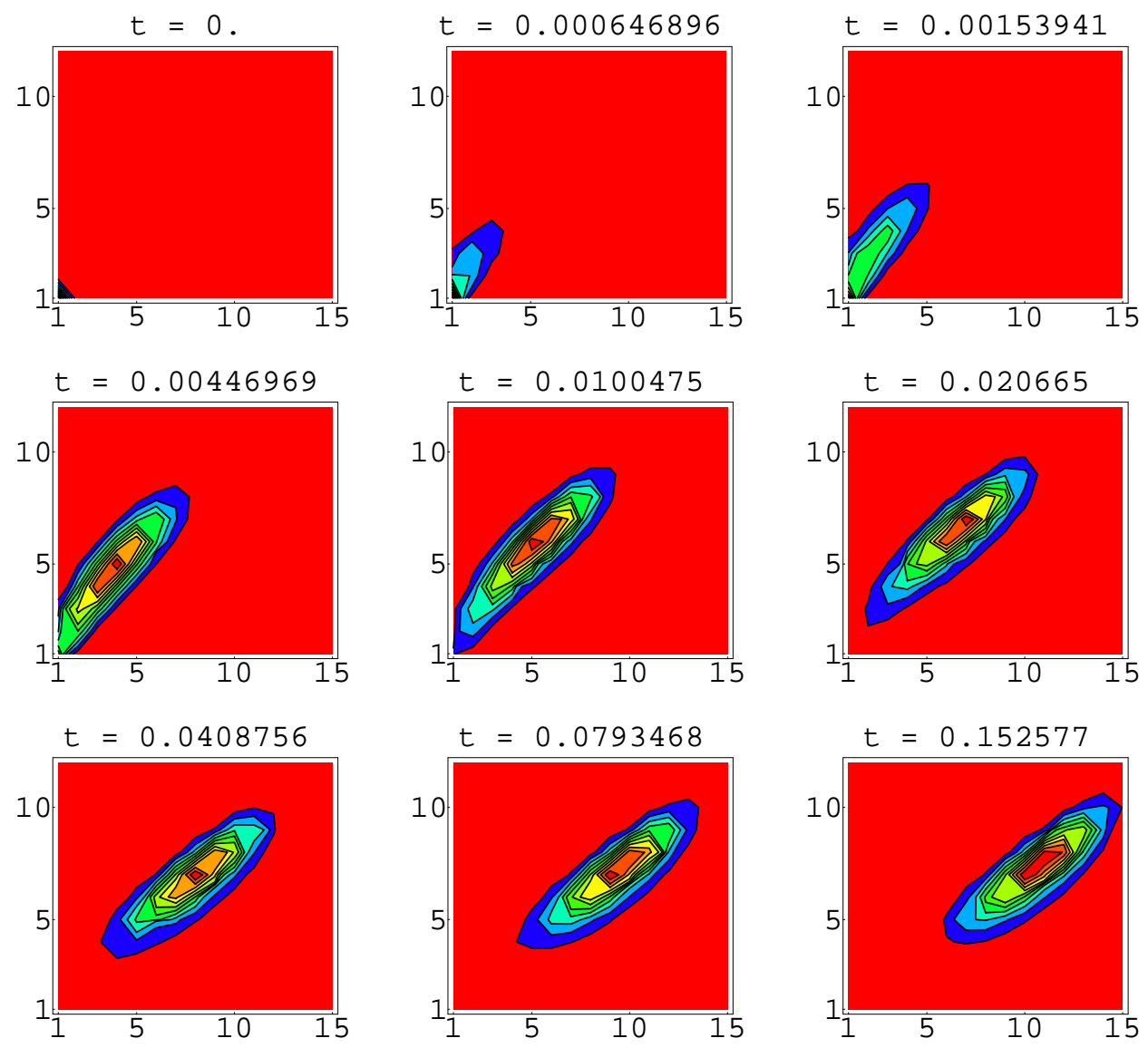

Figure 6: Temporal evolution of for $K=1.1410^{-9}, u_{0}=510^{-7}(T=2300 K)$. The gray levels refer only to the relative values inside one plot. $t$ is the actual time in seconds. Initially all droplets contain 1 enclosure. 


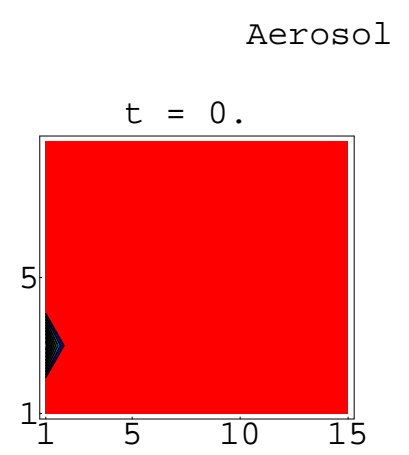

$$
\mathrm{K}=4.26 \cdot 10^{-8} \mathrm{u} 0=5 \cdot \cdot 10^{-7}
$$
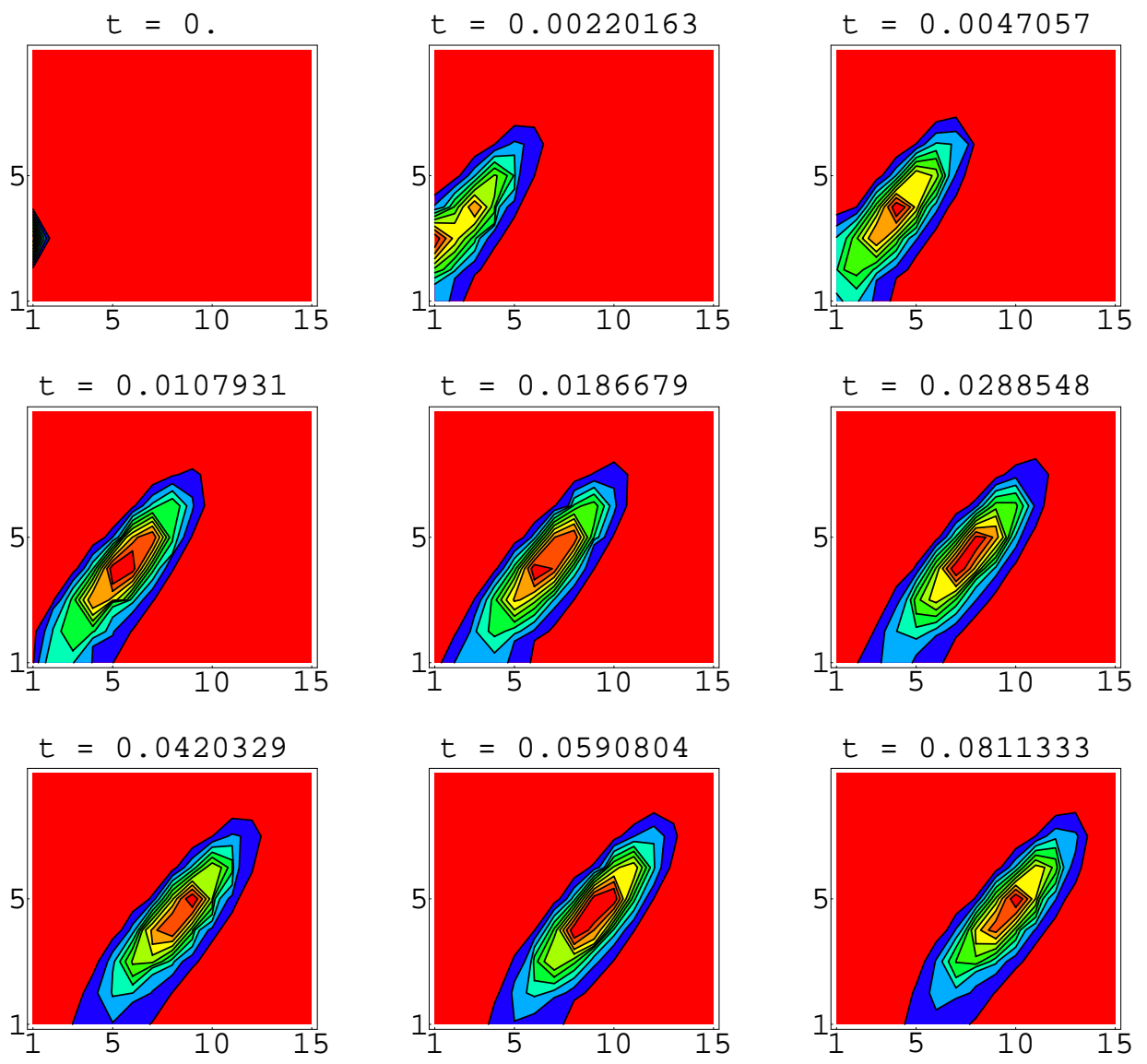

Figure 7: Temporal evolution of for $K=4.2510^{-8}, u_{0}=510^{-7}(T=2300 K)$. The gray levels refer only to the relative values inside one plot. $t$ is the actual time in seconds. 

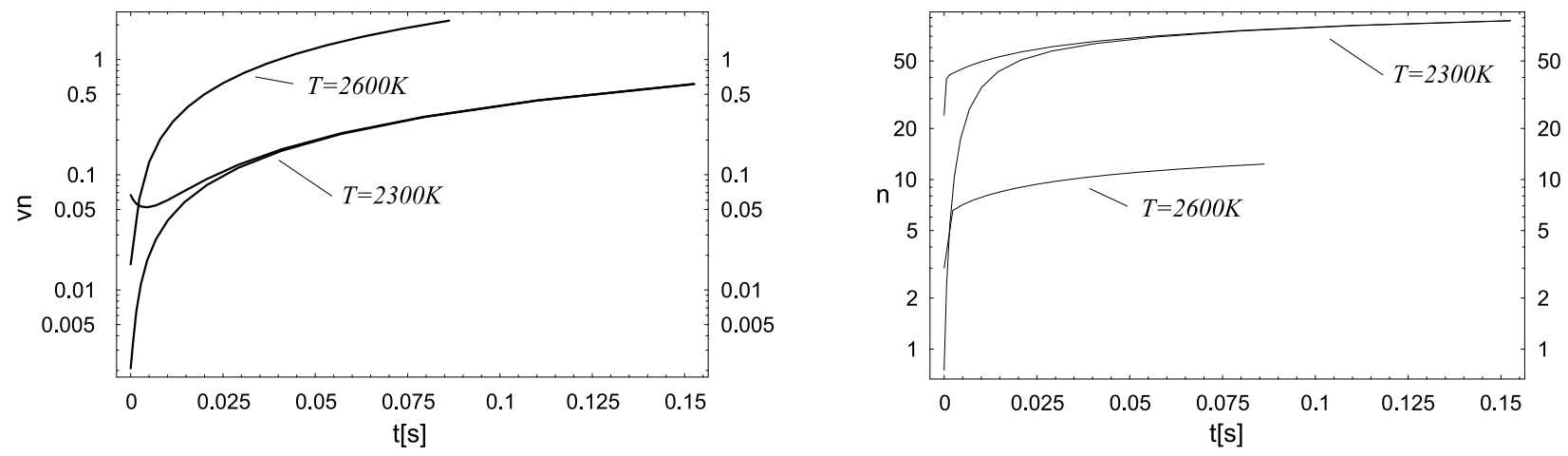

Figure 8: Mean enclosure volume (left, in fractions of the initial droplet size) and mean number of enclosures (right) for the three processes.

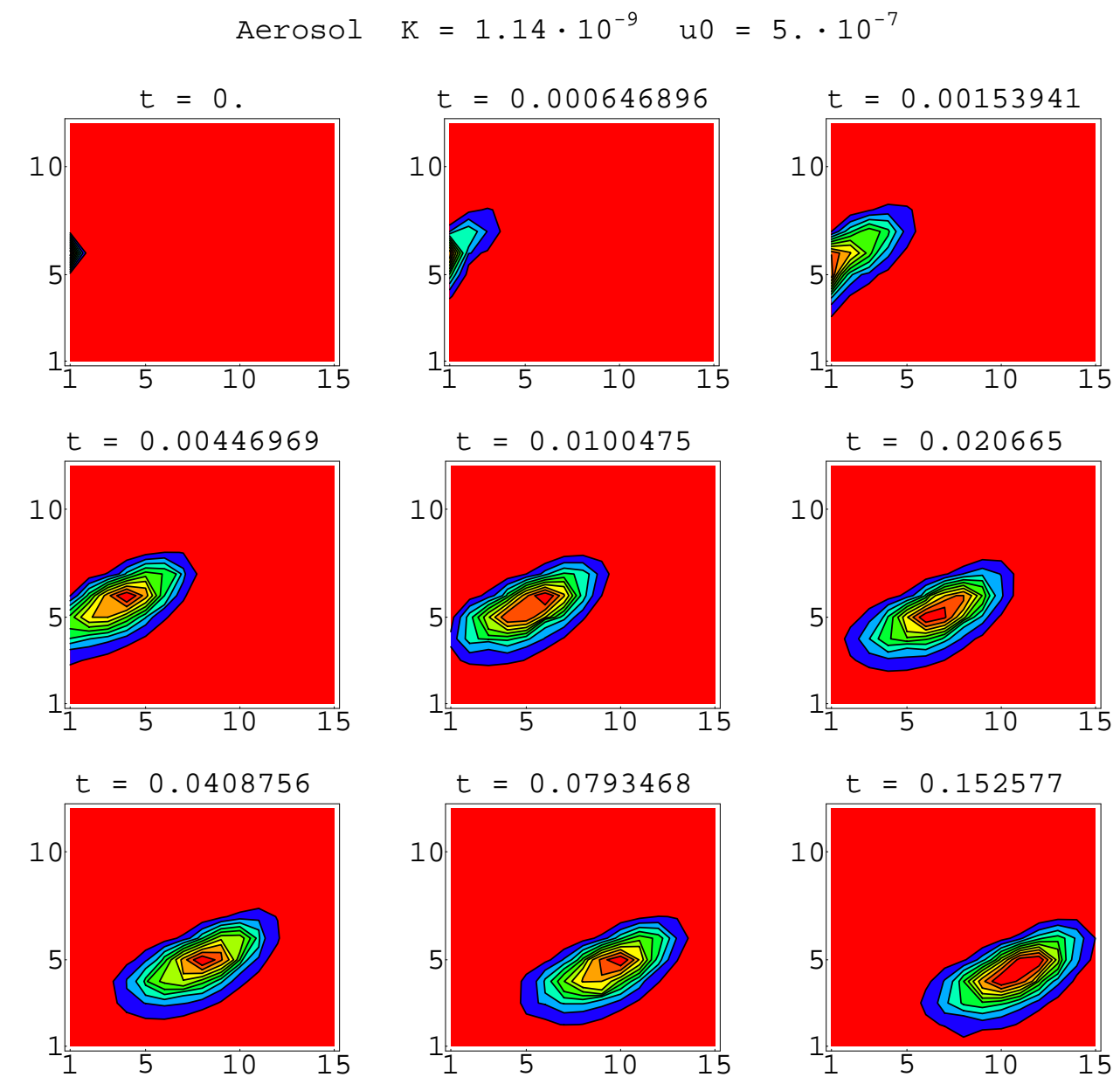

Figure 9: Temporal evolution of $K=1.1410^{-9} u_{0}=510^{-7}, t$ in seconds. 


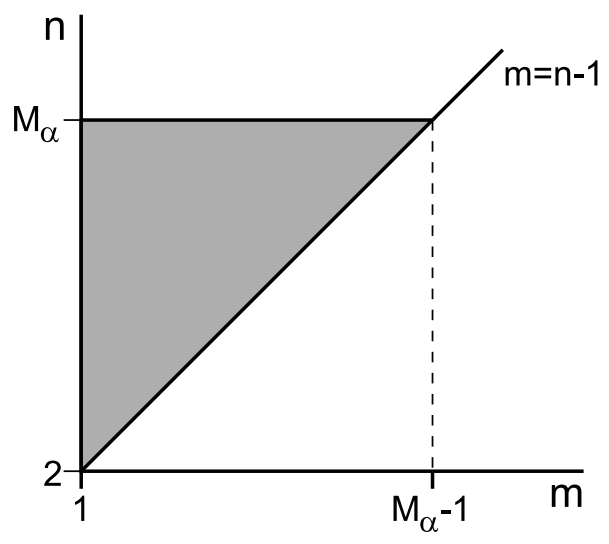

Figure 10: Calculation of $s_{\alpha}$ : All points in the gray area appear in the summation.

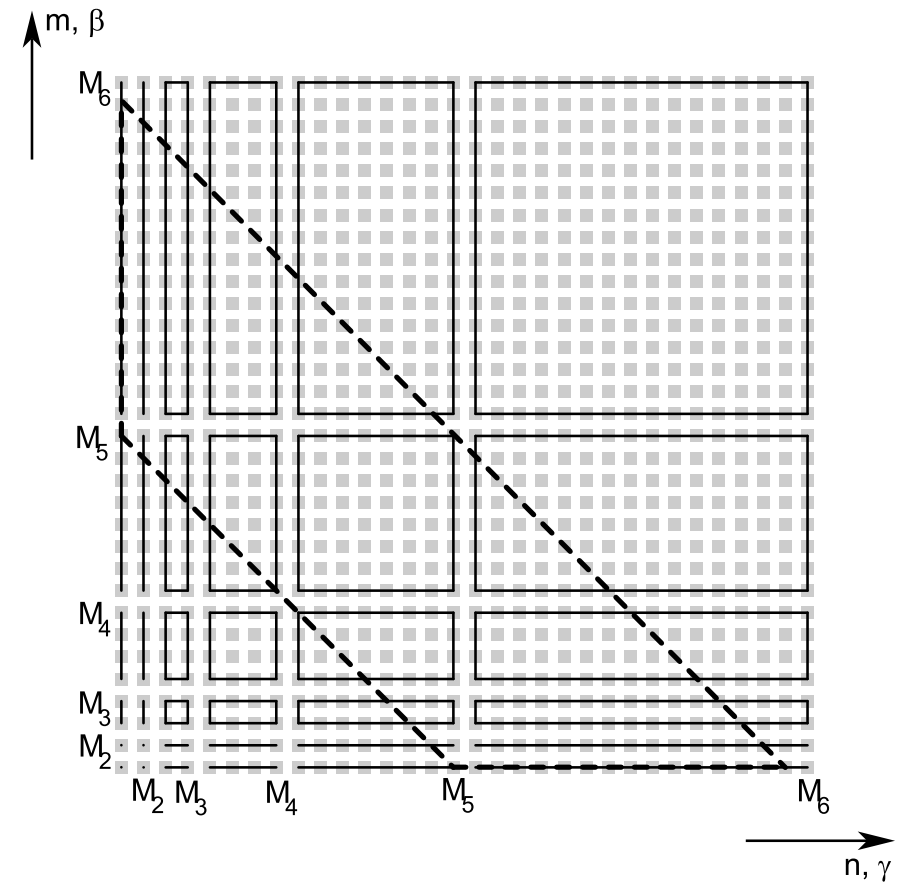

Figure 11: Computation of $S_{6}$, see text for description. 


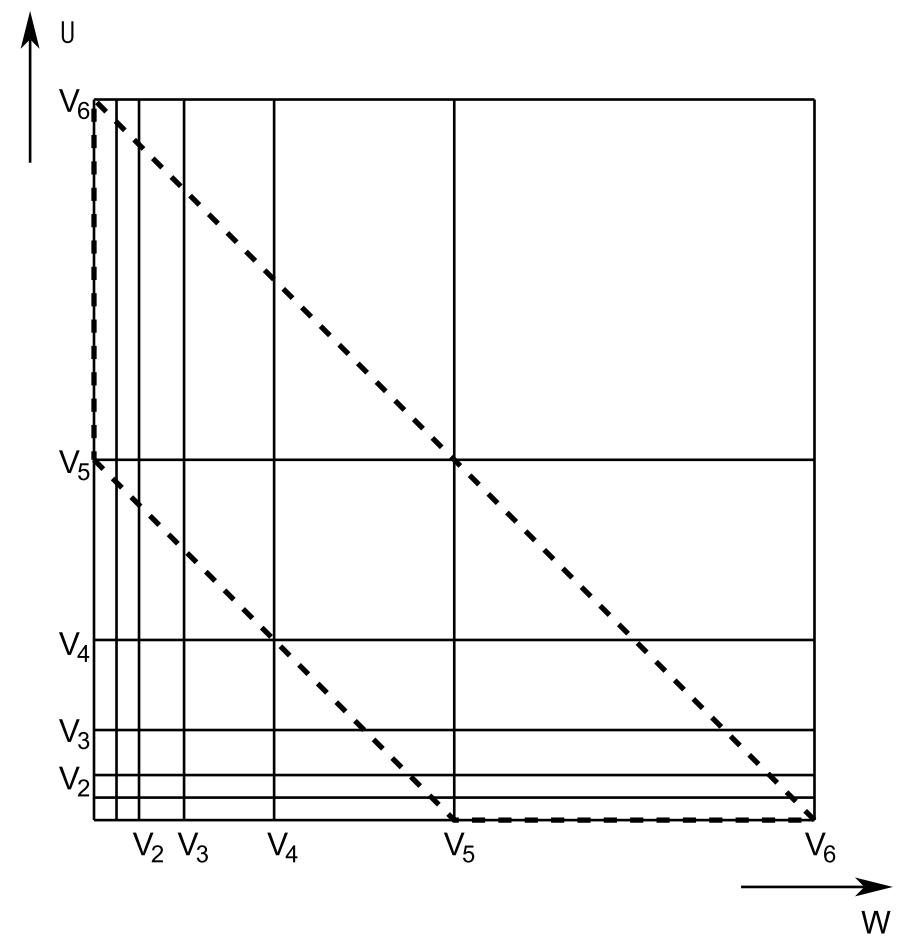

Figure 12: Calculation of $I_{\alpha \beta}^{A}$, see text for details. 